\title{
Synthesis of Phase-Only Reconfigurable Linear Arrays Using Multiobjective Invasive Weed Optimization Based on Decomposition
}

\author{
Yan Liu, ${ }^{1}$ Yong-Chang Jiao, ${ }^{1}$ Ya-Ming Zhang, ${ }^{2}$ and Yan-Yan Tan $^{3}$ \\ ${ }^{1}$ National Key Laboratory of Antennas and Microwave Technology, Xidian University, Xian 710071, China \\ ${ }^{2}$ School of Electronics and Information, Northwestern Polytechnical University, Xian 710072, China \\ ${ }^{3}$ School of Information Science and Engineering, Shandong Normal University, Jinan 250014, China \\ Correspondence should be addressed to Yan Liu; liuyan_xidian@163.com
}

Received 24 April 2014; Revised 25 August 2014; Accepted 26 August 2014; Published 20 October 2014

Academic Editor: Stefano Selleri

Copyright (C) 2014 Yan Liu et al. This is an open access article distributed under the Creative Commons Attribution License, which permits unrestricted use, distribution, and reproduction in any medium, provided the original work is properly cited.

\begin{abstract}
Synthesis of phase-only reconfigurable array aims at finding a common amplitude distribution and different phase distributions for the array to form different patterns. In this paper, the synthesis problem is formulated as a multiobjective optimization problem and solved by a new proposed algorithm MOEA/D-IWO. First, novel strategies are introduced in invasive weed optimization (IWO) to make original IWO fit for solving multiobjective optimization problems; then, the modified IWO is integrated into the framework of the recently well proved competitive multiobjective optimization algorithm MOEA/D to form a new competitive MOEA/D-IWO algorithm. At last, two sets of experiments are carried out to illustrate the effectiveness of MOEA/D-IWO. In addition, MOEA/DIWO is compared with MOEA/D-DE, a new version of MOEA/D. The comparing results show the superiority of MOEA/D-IWO and indicate its potential for solving the antenna array synthesis problems.
\end{abstract}

\section{Introduction}

In actual applications such as satellite communications and radar navigations, single antenna array is generally required to have the capability of producing a number of radiation patterns with different shapes, so as to save space and reduce cost. In practice, changing excitation phases is much easier than changing excitation amplitudes in the feeding network. Keeping this in mind, the phase-only reconfigurable array is designed to radiate multiple radiation patterns using a single power divider network and different phase shifters. Thanks to its advantages, phase-only reconfigurable array attracts much more attentions in recent years [1-10].

During the past decades, a number of design methods for synthesizing phase-only reconfigurable arrays have been proposed [1-10]. These methods can be divided into two categories. One is local search algorithms, like "alternating projections" method [1-3], which are efficient and simple, but sensitive to initial values. The other is the evolutionary algorithms, such as genetic algorithm (GA) [4-6], particle swarm optimization (PSO) [6-8], and differential evolution (DE) $[9,10]$, which have global searching ability. It has been shown that the evolutionary algorithms are more effective and flexible for synthesizing phase-only reconfigurable arrays [4-10]. With the rapid development of high-speed computer technology, more researchers prefer to adopt evolutionary algorithms for designing the pattern reconfigurable arrays.

For pattern reconfigurable arrays, multiple patterns should meet their design indexes simultaneously, and the phase-only synthesis problem can be expressed as a typical multiobjective optimization problem with two or more conflicting objectives.

In most existing literatures, multiple objectives are usually summed into a single objective function with different weights, and the multiobjective optimization problem is converted into a single-objective optimization problem. Although the optimization problem is solved easily by using this approach, some problems arise inevitably. The weights for 
different objectives depend on experience or lots of experiments; thus, decision-makers must have enough prior knowledge of the problem. Only when the weights are set properly can the desired patterns be achieved. In order to achieve more optimal solutions, more experiments need to be done. This would be a very complex and time-consuming process. Hence, in this paper, design of phase-only reconfigurable arrays will be formulated as a multiobjective optimization problem (MOP).

In 2007, Zhang and Li [11] proposed a novel method for MOPs, called multiobjective evolutionary algorithm based on decomposition (MOEA/D), which makes use of the decomposition methods in mathematics and the optimization paradigm in evolutionary computation. Later, a version of MOEA/D employing the differential evolution (DE) operator named MOEA/D-DE [12-14] was proposed and shown to perform well on the MOPs with complicated Pareto set shapes. Theoretically, any evolutionary operator can be used in MOEA/D for actual problems.

Invasive weed optimization (IWO) is a numerical stochastic optimization algorithm inspired from weed colonization, which was proposed by Mehrabian and Lucas in 2006 [15]. The algorithm imitates seed spatial diffusion, growth, reproduction, and competitive exclusion process of invasive weed. With strong robustness and adaptability, IWO converges to the optimal solution effectively. It has been proved that IWO can be used to successfully solve many single-objective optimization problems [16-20] and some MOPs [21].

In order to solve the design problem of phase-only reconfigurable arrays effectively, a new version of MOEA/D called MOEA/D-IWO is proposed. First, in order to improve the performance of IWO in MOEA/D-IWO, we present an adaptive standard deviation, which changes not only with the increase of evolution generations, but also with the cost function value of each individual. This strategy improves the convergence rate and helps the seeds escape from local optimum. Then, the modified IWO with an adaptive standard deviation is integrated into the framework of MOEA/D for solving multiobjective problems. Then, MOEA/D-IWO is formed. Taking advantage of the powerful searching ability of invasive weeds, the overall performance of the proposed MOEA/D-IWO is shown in solving the synthesis problems. The problems are also solved by the existing MOEA/D-DE. The superiority of MOEA/D-IWO over MOEA/D-DE in solving array synthesis problems is demonstrated.

The remainder of this paper is organized as follows. Section 2 describes the definitions of multiobjective optimization problems. Section 3 introduces implementation steps of MOEA/D-IWO. In Section 4, basic principle of pattern reconfigurable arrays is presented. In Section 5, MOEA/D-IWO is used to design the phase-only reconfigurable linear array. Experimental results and discussion are given in Section 6. Finally, Section 7 concludes this paper.

\section{Multiobjective Problems}

Assuming minimizing $M$ objectives simultaneously, a multiobjective optimization problem (MOP) can be stated as follows:

$$
\begin{array}{ll}
\text { Minimize } & \vec{F}(\vec{x})=\left(f_{1}(\vec{x}), f_{2}(\vec{x}), \ldots, f_{M}(\vec{x})\right)^{T} \\
\text { subject to } & \vec{x} \in \Omega,
\end{array}
$$

where $\Omega$ is the decision space, $\vec{x}=\left(x_{1}, \ldots, x_{N}\right)^{T}$ is the decision vector, $F: \Omega \rightarrow R^{M}$ consists of $M$ real-valued objective functions, and $R^{M}$ is called the objective space. The attainable objective set is defined as the set $\{F(x) \mid x \in \Omega\}$. Being different from single-objective optimization, one best solution (global minimum) with respect to all objectives may not exist in MOPs. All the objectives in (1) are treated with the same importance and generally no point in $\Omega$ can minimize all the objectives simultaneously. The aim of MOPs is to find a set of points which cannot be improved in one objective without impairing at least one other objective. These points are called the Pareto solution.

Definition 1 (Pareto dominance). Let $\vec{u}=\left(u_{1}, \ldots, u_{M}\right)^{T}$ and $\vec{v}=\left(v_{1}, \ldots, v_{M}\right)^{T} \in R^{M}$ be two vectors; $u$ is said to dominate $v$, denoted by $u<v$, if

$$
\forall i \in\{1, \ldots, M\}, \quad u_{i} \leq v_{i} \wedge \exists j \in\{1, \ldots, M\}, \quad u_{j}<v_{j} .
$$

Note that if Pareto dominance does not hold between two vectors, then they are considered nondominated with each other.

Definition 2 (Pareto optimality). $\vec{x}_{u} \in \Omega$ is said to be Pareto optimal, if and only if

$$
\nexists \vec{x}_{v} \in \Omega: \vec{F}\left(\vec{x}_{v}\right)<\vec{F}\left(\vec{x}_{u}\right) .
$$

Definition 3 (Pareto optimal set). The Pareto optimal set, denoted by PS, is defined as

$$
\text { PS : }=\left\{\vec{x}_{u} \in \Omega \mid \nexists \vec{x}_{v} \in \Omega: \vec{F}\left(\vec{x}_{v}\right)<\vec{F}\left(\vec{x}_{u}\right)\right\} .
$$

Definition 4 (Pareto front). For a given MOP $\vec{F}(\vec{x})$ and its Pareto optimal set PS, the Pareto front is defined as

$$
\mathrm{PF}:=\left\{\vec{u}=\vec{F}\left(\vec{x}_{u}\right) \mid \vec{x}_{u} \in \mathrm{PS}\right\} .
$$

Instead of searching for a single or just a few optimal solutions as in solving single-objective problems, the goal of handling multiobjective problem is to find the Pareto front as well as the Pareto set of the problem.

\section{The Main Procedures of MOEA/D-IWO}

One of the key issues of MOEA/D is that a decomposition method is used to transform an MOP into a number of 
single-objective optimization subproblems, and MOEA/D optimizes these subproblems collectively and simultaneously. The objective function of each single-objective subproblem is an aggregation of all the objective functions in MOP. At each generation, the population is composed of the best solutions found so far for each subproblem, which is optimized by using information only from its neighboring subproblems. Neighborhood relations among these singleobjective subproblems are defined based on the Euclidian distances between their weight vectors. MOEA/D exploits information sharing among subproblems which are neighbors to accomplish the optimization task effectively and efficiently. MOEA/D-IWO maintains the basic framework of MOEA/D [11], and the IWO algorithm is used for producing new solutions. When MOEA/D-IWO is used to optimize MOP, the following issues need to be considered.

3.1. Produce the Weight Vectors. As in [11], we use the simplexlattice design method [22] to produce the weight vectors, in which each individual weight takes value from $\{0 / H$, $1 / H, \ldots, H / H\}$, where $H$ is an integer.

3.2. Decompose an MOP into a Number of SingleObjective Subproblems. In theory, weighted sum, Tchebycheff approach, boundary intersection, and any other decomposition approach can be used to convert an MOP into a number of single-objective subproblems [11]. Tchebycheff approach is mainly employed in our experimental study. Let $\lambda^{1}, \lambda^{2}, \ldots, \lambda^{P}$ be a set of even spread $P$ weight vectors, where $\lambda^{i}=\left(\lambda_{1}^{i}, \lambda_{2}^{i}, \ldots, \lambda_{M}^{i}\right)^{T}$ is the weight vector for the $i$ th subproblem, $\lambda_{j}^{i} \geq 0, j=1,2, \ldots, M$, and $\sum_{j=1}^{M} \lambda_{j}^{i}=1, M$ is the number of objectives in (1), $i=1,2, \ldots, P . z^{*}$ represents the reference point. The $i$ th single-objective optimization subproblem obtained by decomposing the given MOP can be represented as [23]

$$
\begin{aligned}
& \text { Minimize } g^{t e}\left(x \mid \lambda^{i}, z^{*}\right)=\max _{1 \leq j \leq M}\left\{\lambda_{j}^{i}\left|f_{j}(x)-z_{j}^{*}\right|\right\} \\
& \text { subject to } \quad x \in \Omega .
\end{aligned}
$$

The Tchebycheff approach searches solutions through minimizing $g^{\text {te }}$ toward $z^{*}$, which has an advantage that both convex and concave Pareto front can be approximated [24]. All these $P$ single-objective functions are optimized simultaneously in each run. If we use a large $P$ and select the weight vectors properly, all the optimal solutions of the single-objective problems from decomposition will well approximate the Pareto front. The optimization process is accomplished by the collaboration of the neighbors for each subproblem.

\subsection{Confirm the Neighbors of Each Single-Objective Sub-} problem. In MOEA/D, each subproblem is optimized by using information mainly from its neighboring subproblems. The neighborhood relations among these subproblems are defined based on the distances between their weight vectors. Compute the Euclidean distances between any two weight vectors and then work out the $T$ closest weight vectors to each weight vector. For the $i$ th subproblem, its neighbor is set to $B(i)=\left\{i_{1}, i_{2}, \ldots, i_{T}\right\}, i=1,2, \ldots, P$, where $\lambda^{i_{1}}, \lambda^{i_{2}}, \ldots, \lambda^{i_{T}}$ are $T$ weight vectors close to $\lambda^{i}$. Single-objective subproblems, which are considered neighbors, will have similar fitness landscapes and their optimal solutions should be close in the decision space. MOEA/D exploits information sharing among subproblems, which are neighbors, to accomplish the optimization task effectively and efficiently.

3.4. The Selection of Evolution Strategy. The IWO operator is integrated into MOEAD-IWO to produce new solutions. In IWO, new solutions (seeds) produced are randomly distributed in $D$-dimensional space around their parents (weed) in normal distribution $N\left(0, \sigma_{\text {iter }}^{2}\right)$; the change rule for $\sigma_{\text {iter }}$ is expressed as

$$
\sigma_{\text {iter }}=\sigma_{\text {final }}+\left(\frac{\text { iter }_{\text {max }}-\text { iter }}{\text { iter }_{\max }}\right)^{\text {pow }}\left(\sigma_{\text {initial }}-\sigma_{\text {final }}\right),
$$

where $\sigma_{\text {initial }}$ and $\sigma_{\text {final }}$ are initial and final standard deviations and pow is the nonlinear regulatory factor. It can be seen from (7) that $\sigma_{\text {iter }}$ decreases with the increase of evolution generations, while the $\sigma_{\text {iter }}$ value of each subproblem in one generation is the same. This is not conducive to the algorithm convergence. Thus, we modify IWO and present an adaptive standard deviation, in which the value of $\sigma_{\text {iter }}$ in one generation changes not only with the cost function value of each subproblem but also with the maximum and minimum cost function values in the generation, as shown in the following equation:

$$
\sigma_{\text {iter }, i}= \begin{cases}\left(1+\gamma \frac{\mathrm{FV}_{i}-\mathrm{FV}_{a}}{\mathrm{FV}_{\max }-\mathrm{FV}_{a}}\right) \sigma_{\text {iter, }}, & \mathrm{FV}_{i} \geq \mathrm{FV}_{a} \\ \left(1-\gamma \frac{\mathrm{FV}_{a}-\mathrm{FV}_{i}}{\mathrm{FV}_{a}-\mathrm{FV}_{\min }}\right) \sigma_{\text {iter }}, & \mathrm{FV}_{i}<\mathrm{FV}_{a}\end{cases}
$$

where $\mathrm{FV}_{i}$ is the cost function value of the $i$ th subproblem, $\mathrm{FV}_{\text {max }}, \mathrm{FV}_{\text {min }}$, and $\mathrm{FV}_{a}$ denote the maximum, minimum, and average cost function values among all these subproblems, respectively, and $\gamma$ is the zoom factor controlling the variation range of the standard deviation, which takes value from 0 to 1. In this paper, we take $\gamma=0.5$.

It can be seen that, for the minimization problem, the subproblems with lower cost function values have smaller standard deviation in one generation, which enables the seeds distributed around the better subproblems. Moreover, the range of the adaptive standard deviation $\sigma_{\text {iter, } i}$ is [1 $\gamma, 1+\gamma] \sigma_{\text {iter }}$, which makes the standard deviation of one subproblem in the younger generation likely to be larger than that in the older generation. This will help the new produced seeds escape from local optimum, improve the convergence rate, and balance the global and local search capabilities effectively at the same time.

In the present generation, the number of seeds produced by the $i$ th subproblem is calculated by

$$
s_{\text {num }}=\text { floor }\left(\frac{\mathrm{FV}_{\text {max }}-\mathrm{FV}_{i}}{\mathrm{FV}_{\text {max }}-\mathrm{FV}_{\text {min }}}\left(s_{\text {max }}-s_{\text {min }}\right)+s_{\text {min }}\right) \text {, }
$$


where $s_{\max }$ and $s_{\min }$ are the largest and smallest numbers of seeds produced, respectively, and floor() represents the round-down function. It is clear that better population members produce more seeds.

Suppose $x^{i}=\left(x_{1}^{i}, x_{2}^{i}, \ldots, x_{N}^{i}\right)^{T}, i=1,2, \ldots, P$, is the present optimal solution for the $i$ th subproblem, and each new solution produced by $x^{i}$ is $y=\left(y_{1}, y_{2}, \ldots, y_{N}\right)^{T}$, in which each element $y_{k}$ is generated as follows:

$$
y_{k}=x_{k}^{i}+N\left(0, \sigma_{\text {iter }, i}^{2}\right), \quad k=1,2, \ldots, N .
$$

Then $s_{\text {num }}$ new solutions are used to update the $i$ th subproblem and its neighborhoods.

3.5. Metrics for Comparing Nondominated Sets. Several metrics have been proposed to compare the performance of different multiobjective evolutionary algorithms quantitatively, such as generational distances (GD) [25], inverted generational distance (IGD) [26], and spread [27]. When using the above three metrics, we need to know the true Pareto front of the optimization problem first. However, in actual engineering problem, we cannot obtain the true Pareto front [28]. Then, in this section, three metrics are adopted to make a quantitative assessment of the performance of MOEA/D-DE and MOEA/D-IWO.

Before giving the performance metrics, we first present normalization of vectors in objective space. Assume we have $p$ nondominated sets, $Z_{1}, Z_{2}, \ldots, Z_{p}$. First we set $Z=\left\{Z_{i}, i=\right.$ $1,2, \ldots, p\}$. Then, we define $f_{i}^{\min }=\min \left\{f_{i}(\bar{x}), F(\bar{x})=z \in Z\right\}$ and $f_{i}^{\max }=\max \left\{f_{i}(\bar{x}), F(\bar{x})=z \in Z\right\}$, which correspond to the minimum and maximum values for the $i$ th coordinate of the objective space. Then, for all coordinates, $i=1,2, \ldots, n$, we calculate all points according to

$$
\bar{f}_{i}(\bar{x})=\frac{f_{i}(\bar{x})-f_{i}^{\min }}{f_{i}^{\max }-f_{i}^{\min }} .
$$

The normalized vector of a vector $z$ is denoted by $\bar{z}$; the set of normalized vectors is shown as $\bar{Z}$.

3.5.1. D-Metric for Accuracy [29]. Suppose we have two normalized nondominated sets $\bar{Z}_{1}$ and $\bar{Z}_{2} ; \forall \bar{a} \in \bar{Z}_{1}$, we search for $\exists \bar{b} \in \bar{Z}_{21} \subseteq \bar{Z}_{2}$ such that $\bar{b}<\bar{a}$. Then we calculate Euclidean distances from $\bar{a}$ to all points $\bar{b} \in \bar{Z}_{21}$. So the $D$ metric is defined as

$$
D\left(\bar{Z}_{1}, \bar{Z}_{2}\right)=\sum_{\bar{a} \in \bar{Z}_{1}} \frac{\|\bar{a}-\bar{b}\|_{\max }}{\sqrt{n}\left|\bar{Z}_{1}\right|},
$$

where $\|\bar{a}-\bar{b}\|_{\max }=\max \left\{\|\bar{a}-\bar{b}\|, \bar{a} \in \bar{Z}_{1}, \bar{b} \in \bar{Z}_{21}\right\}$ and $n$ is the dimension of the objective space. The $D$-metric returns a value among 0 and 1 , where the smaller, the better. The maximum distance from a dominating point is taken as a basis for accuracy.

3.5.2. $\Delta$-Metric for Uniformity [29]. For a given normalized nondominated set $\bar{Z}$, we define $d_{i}$ to be the Euclidean distance between two consecutive vectors, $i=1,2, \ldots,(|\bar{Z}|-1)$. Let $\widehat{d}=\sum_{i=1}^{|\bar{Z}|-1} d_{i} /(|\bar{Z}|-1)$.Then we define

$$
\Delta(\bar{Z})=\sum_{i=1}^{|\bar{Z}|-1} \frac{\left|d_{i}-\hat{d}\right|}{\sqrt{n}(|\bar{Z}|-1)} .
$$

Note that $0 \leq \Delta(\bar{Z}) \leq 1$ where 0 is the best.

3.5.3. $\nabla$-Metric for Extent [29]. Given a nondominated $\operatorname{set} Z$, we define

$$
\nabla(Z)=\prod_{i=1}^{n}\left|f_{i}^{\max }-f_{i}^{\min }\right|,
$$

where $n$ is the dimension of the objective space. A bigger value spans a larger portion of the hypervolume and therefore is always better.

\section{Basic Principle of Pattern Reconfigurable Array Antennas}

Design of phase-only reconfigurable antenna array aims at finding a common amplitude distribution and different phase distributions, such that the array can produce multiple different patterns.

Consider a linear equispaced array with $N$ elements. If $M$ different patterns need to be produced only by changing the excitation phases of the array under the common excitation amplitude distribution, the optimization variable $x$ is a vector with $M N+N$ elements, where $x_{n}(n=1,2, \ldots, N)$ is the excitation amplitude for the $n$th antenna element denoted by $I_{n}$ and $x_{m N+n}(m=1,2, \ldots, M, n=1,2, \ldots, N)$ is the excitation phase for the $n$th antenna element and the $m$ th pattern, denoted by $\varphi_{m n}$. Then, the complex excitation of the $n$th element in the $m$ th pattern is

$$
i_{m n}=I_{n} \cdot e^{j \varphi_{m n}}=x_{n} e^{j x_{n+m N}} .
$$

It can be seen from (15) that, in the process of optimization, the common excitation amplitude is used for $M$ patterns all the time, and only phases of the excitation are different. The $m$ th pattern produced by the antenna array for far field is given by

$$
F_{m}(u)=\frac{\operatorname{EF}(u)}{F_{\max }} \sum_{n=1}^{N} i_{m n} \cdot e^{j 2 \pi n d u / \lambda},
$$

where $m=1,2, \ldots, M, d$ is the spacing between array elements, $\lambda$ is the wavelength in free space, $u=\sin \theta, \theta$ is the angle from ray direction to normal of array axis, $\operatorname{EF}(\theta)$ is element factor $\left(\operatorname{EF}(\theta)=1\right.$ for isotropic source), and $F_{\max }$ is peak value of far field pattern.

In this paper, patterns we need to reconfigure by the phase-only reconfigurable linear arrays are presented below.

(1) A flat-top beam and a pencil beam: the design problem is expressed as

$$
\text { minimize } F(x)=\left(f_{1}(x), f_{2}(x)\right)^{T} .
$$


Table 1: Design objectives and simulated results for Problem 1.

\begin{tabular}{|c|c|c|c|c|c|c|}
\hline \multirow{2}{*}{ Design parameters } & \multicolumn{3}{|c|}{ Flat-top beam } & \multicolumn{3}{|c|}{ Pencil beam } \\
\hline & Desired & $\begin{array}{c}\text { Obtained by } \\
\text { MOEA/D-DE }\end{array}$ & $\begin{array}{l}\text { Obtained by } \\
\text { MOEA/D-IWO }\end{array}$ & Desired & $\begin{array}{c}\text { Obtained by } \\
\text { MOEA/D-DE }\end{array}$ & $\begin{array}{l}\text { Obtained by } \\
\text { MOEA/D-IWO }\end{array}$ \\
\hline $\begin{array}{l}\text { Side lobe level } \\
\text { (SLL, in } \mathrm{dB})\end{array}$ & -25.00 & -25.46 & -26.13 & -30.00 & -29.56 & -30.50 \\
\hline $\begin{array}{l}\text { Half-power beam width } \\
\text { (HPBW, in } \theta \text {-space) }\end{array}$ & $24^{\circ}$ & $24.2^{\circ}$ & $24^{\circ}$ & $6.8^{\circ}$ & $6.8^{\circ}$ & $6.8^{\circ}$ \\
\hline $\begin{array}{l}\text { Beam width at SLL } \\
\text { (BW, in } \theta \text {-space) }\end{array}$ & $40^{\circ}$ & $41.6^{\circ}$ & $40.2^{\circ}$ & $20^{\circ}$ & $20^{\circ}$ & $20^{\circ}$ \\
\hline $\begin{array}{l}\text { Ripple } \\
\left(\text { in } \mathrm{dB}, 78^{\circ} \leq \theta \leq 102^{\circ}\right)\end{array}$ & 0.50 & 0.50 & 0.49 & N/A & N/A & $\mathrm{N} / \mathrm{A}$ \\
\hline
\end{tabular}

TABLe 2: Design objectives and simulated results for Problem 2.

\begin{tabular}{|c|c|c|c|c|c|c|}
\hline \multirow{2}{*}{ Design parameters } & \multicolumn{3}{|c|}{ Flat-top beam } & \multicolumn{3}{|c|}{ Cosecant-squared beam } \\
\hline & Desired & $\begin{array}{l}\text { Obtained by } \\
\text { MOEA/D-DE }\end{array}$ & $\begin{array}{c}\text { Obtained by } \\
\text { MOEA/D-IWO }\end{array}$ & Desired & $\begin{array}{l}\text { Obtained by } \\
\text { MOEA/D-DE }\end{array}$ & $\begin{array}{c}\text { Obtained by } \\
\text { MOEA/D-IWO }\end{array}$ \\
\hline $\begin{array}{l}\text { Side lobe level } \\
\text { (SLL, in } \mathrm{dB})\end{array}$ & -25.00 & -24.0106 & -24.9659 & -25.00 & -24.0735 & -24.9060 \\
\hline $\begin{array}{l}\text { Beam width at SLL } \\
\text { (BW, in } \theta \text {-space) }\end{array}$ & 0.84 & 0.88 & 0.86 & 0.84 & 0.88 & 0.86 \\
\hline $\begin{array}{l}\text { Ripple } \\
\text { (in dB) }\end{array}$ & $\begin{array}{c}0.60 \\
|u| \leq 0.32\end{array}$ & 0.5998 & 0.5994 & $\begin{array}{c}0.60 \\
0 \leq u \leq 0.64\end{array}$ & 0.8588 & 0.5341 \\
\hline
\end{tabular}

The cost functions of each single pattern in (17) are

$$
\begin{aligned}
& f_{1}(x)=\text { Cost }_{f}=\sum_{s=1}^{4}\left(\max \left\{Q_{s, d}-Q_{s}(x), 0\right\}\right)^{2}, \\
& f_{2}(x)=\text { Cost }_{p}=\sum_{s=1}^{3}\left(\max \left\{Q_{s, d}-Q_{s}(x), 0\right\}\right)^{2} .
\end{aligned}
$$

(2) A flat-top beam and a cosecant beam: the design problem is expressed as

$$
\text { minimize } F(x)=\left(f_{3}(x), f_{4}(x)\right)^{T} \text {. }
$$

The cost functions of each single pattern in (20) are

$$
\begin{aligned}
& f_{3}(x)=\operatorname{Cost}_{f}=\sum_{s=1}^{3}\left(\max \left\{Q_{s, d}-Q_{s}(x), 0\right\}\right)^{2}, \\
& f_{4}(x)=\text { Cost }_{c}=\sum_{s=1}^{3}\left(\max \left\{Q_{s, d}-Q_{s}(x), 0\right\}\right)^{2},
\end{aligned}
$$

where $\operatorname{Cost}_{f}$, Cost ${ }_{c}$, and Cost ${ }_{p}$ represent the cost functions of the flat-top beam, cosecant-squared beam, and pencil beam, respectively, and $Q_{s, d}$ and $Q_{s}(x)$ are the desired and calculated values for each design specification we use. Desired and calculated values of each design index in (18), (19), (21), and (22) are summarized in Tables 1 and 2. The lower the cost value is, the closer the calculated value approaches the desired value. When the calculated values of all the indexes are less than the corresponding desired values, the cost value is set to zero.

\section{MOEA/D-IWO for Synthesis of Phase-Only Reconfigurable Linear Array}

Suppose $P$ is the population size; that is to say, we need to optimize $P$ subproblems in a single run; $\left\{\lambda^{1}, \lambda^{2}, \ldots, \lambda^{P}\right\}$ is a set of weight vectors. The objective functions of all the patterns are summed with assigned weights. For each singleobjective subproblem, the objective function is described as follows:

$$
g^{t e}\left(x \mid \lambda^{i}, z^{*}\right)=\max _{1 \leq m \leq M}\left\{\lambda_{m}^{i}\left|f_{m}(x)-z_{m}^{*}\right|\right\}
$$

where $\lambda^{i}=\left(\lambda_{1}^{i}, \lambda_{2}^{i}, \ldots, \lambda_{M}^{i}\right)^{T}, i=1,2, \ldots, P$. MOEAD-IWO is used to minimize $P$ objective functions (23) simultaneously in a single run, and the neighborhood of $\lambda^{i}$ is the several weight vectors close to $\lambda^{i}$.

At each generation, MOEAD-IWO maintains the following information:

(i) a population of $P$ points $x^{1}, x^{2}, \ldots, x^{P} \in \Omega$, where $\Omega$ is the feasible solution space of the excitation amplitude and phases;

(ii) $\mathrm{FV}^{1}, \mathrm{FV}^{2}, \ldots, \mathrm{FV}^{P}$, where $\mathrm{FV}^{i}=g^{t e}\left(x^{i} \mid \lambda^{i}\right), i=$ $1,2, \ldots, P$

(iii) Gen: the current generation number;

(iv) $z=\left(z_{1}, z_{2}, \ldots, z_{M}\right)^{T}$, where $z_{m}$ is the best value found so far for objective $f_{m}$. 
The algorithm is outlined as follows.

Input

(i) The multiobjective problem (17) or (20).

(ii) A stopping criterion.

(iii) $P$ : the population size (the number of subproblems).

(iv) $T$ : the number of the neighbors for each subproblem.

(v) A uniform set of $P$ weight vectors $\lambda^{1}, \lambda^{2}, \ldots, \lambda^{P}$.

Output

(i) Approximation to the PS: $x^{1}, x^{2}, \ldots, x^{P}$.

(ii) Approximation to the PF: $F\left(x^{1}\right), F\left(x^{2}\right), \ldots, F\left(x^{P}\right)$.

Step 1 (initialization). Consider the following.

Step 1.1. Generate the weight vectors $\lambda^{1}, \lambda^{2}, \ldots, \lambda^{P}$.

Step 1.2. Compute the Euclidian distances between any two weight vectors to each weigh vector. For each $i=1,2, \ldots, P$, set $B(i)=\left\{i_{1}, i_{2}, \ldots, i_{T}\right\}$, where $\lambda^{i_{1}}, \lambda^{i_{2}}, \ldots, \lambda^{i_{T}}$ are $T$ weight vectors close to $\lambda^{i}$.

Step 1.3. Generate an initial population $x^{1}, x^{2}, \ldots, x^{P}$ by uniformly random sampling from the search space $\Omega$.

Set $\mathrm{FV}^{i}=g^{t e}\left(x^{i} \mid \lambda^{i}\right)$.

Step 1.4. Initialize $z=\left(z_{1}, z_{2}, \ldots, z_{M}\right)^{T}$ by setting $z_{m}=$ $\min _{1 \leq i \leq P} f_{m}\left(x^{i}\right)$.

Step 2 (update). For $i=1,2, \ldots, P$, do the following.

Step 2.1 (selection of mating/update range). Generate a number rand from $[0,1]$ randomly. Then set

$$
S= \begin{cases}B(i), & \text { if rand }<\delta, \\ \{1,2, \ldots, P\}, & \text { otherwise }\end{cases}
$$

$\delta$ is the probability that parent individuals are selected from the neighborhood.

Step 2.2 (reproduction). Calculate the number of new solutions $s_{\text {num }}$ using (9) and produce new solutions $y^{k}(k=$ $\left.1,2, \ldots, s_{\text {num }}\right)$ using $(8)$.

Step 2.3 (repair). If an element in $y^{k}$ is out of the boundary of $\Omega$, its value is reset to the boundary value.

Step 2.4 (update of $z$ ). For each $m=1,2, \ldots, M$, if $f_{m}\left(y^{\min }\right)<$ $z_{m}$, in which $y^{\min }$ is the minimum solution among $\left\{y^{k}, k \in\right.$ $\left.\left\{1,2, \ldots, s_{\text {num }}\right\}\right\}$, then set $z_{m}=f_{m}\left(y^{\text {min }}\right)$.

Step 2.5 (update of solutions). Set $c=0$ and then do the following.

(1) If $c=\eta_{r}$ or $S$ is empty, go to Step 3. Otherwise, randomly pick an index $j$ from $S$.
(2) If $\min \left\{g^{t e}\left(y^{1} \mid \lambda^{j}\right), g^{t e}\left(y^{2} \mid \lambda^{j}\right), \ldots, g^{t e}\left(y^{s_{\text {num }}} \mid \lambda^{j}\right)\right\} \leq$ $g^{t e}\left(x^{j} \mid \lambda^{j}\right)$, then set $x^{j}=y^{\min }, \mathrm{FV}^{j}=g^{t e}\left(y^{\min } \mid\right.$ $\left.\lambda^{j}\right)$, in which $y^{\text {min }}$ is the minimum solution among $\left\{y^{k}, k \in\left\{1,2, \ldots, s_{\text {num }}\right\}\right.$. Set $c=c+1$.

(3) Delete $j$ from $S$ and go to (1).

Step 3 (stopping criteria). If stopping criteria are satisfied, then stop and output $\left\{x^{1}, x^{2}, \ldots, x^{P}\right\}$ and $F\left(x^{1}\right), F\left(x^{2}\right)$, $\ldots, F\left(x^{P}\right)$. Otherwise, go to Step 2 .

In the population, optimization variables for each individual are the common excitation amplitudes for all patterns and different phases for forming different patterns. The output of the algorithm is a Pareto set, in which each Pareto optimal solution corresponds to a phase-only reconfigurable array. In the optimization results, different weight coefficients corresponding to various patterns form a weight vector, each Pareto optimal solution corresponds to a weight vector, and different Pareto optimal solutions have different weight vectors. In actual applications, decision-makers select a desired solution from the approximated PF or obey some standards for choosing the best compromise solution.

\section{Experiments Results}

To demonstrate whether the proposed MOEA/D-IWO outperforms MOEA/D-DE in the design of phase-only pattern reconfigurable linear arrays, we carry out two experiments.

6.1. Parameter Setting. In MOEA/D, the population size and weight vectors are controlled by an integer $H . \lambda^{1}, \lambda^{2}, \ldots, \lambda^{P}$ are the weight vectors in which each individual weight takes a value from $\{0 / H, 1 / H, \ldots, H / H\}$; therefore the population size is $P=C_{H+M-1}^{M-1}$, where $M$ is the number of objectives. In the experiments, population size $P$ is set to $201(H=200)$ for 2-objective instances. For the other control parameters, we set $T=0.1 P, \eta_{r}=0.01 P$, and $\delta=0.7$ [30], which are the same for MOEA/D-DE and MOEA/D-IWO. For DE operator, we set the crossover rate $\mathrm{CR}=1.0$, the scalar factor $F=0.5$, $\eta=20$, and $p_{m}=1 / n$ in mutation operators $[12,30]$. For IWO operator, we set $\sigma_{\text {initial }}=0.1, \sigma_{\text {final }}=0.0002$, pow $=$ $3, s_{\max }=3$, and $s_{\min }=1$. To be fair in the comparison, both algorithms stop after reaching the maximum function evaluations 500000 for all the tests. In order to restrict the influence of random effects, both algorithms run 20 times independently of each problem, and the best results are presented in this section. Source codes for MOEA/D-DE are taken from [31].

6.2. Determining the Best Compromise Solution. When facing a set of nondominated solutions, a decision-maker's judgment may have fuzzy or imprecise goals for each objective function, so useful quantitative measures for assessing the quality of each nondominated solution are needed. In present literatures, an effective method based on the concept of fuzzy sets is proposed [25] and successfully used in some MOPs [32-34], in which a membership function $\mu_{i}$ is defined for each objective $f_{i}$ by taking account of the minimum and 
TABLE 3: The best compromise solutions for the first instance by MOEA/D-IWO and MOEA/D-DE (DRR = 4).

\begin{tabular}{lcccc}
\hline & $f_{1}$ (flat-top beam) & $f_{2}$ (pencil beam) & $\left(\lambda_{1}, \lambda_{2}\right)$ & Cost $=\lambda_{1} f_{1}+\lambda_{2} f_{2}$ \\
\hline MOEA/D-DE with TE & 0.0069 & 0.0059 & $(0.5350,0.4650)$ & 0.0064 \\
MOEA/D-DE with WS & 0.0086 & 0.0084 & $(0.5100,0.4900)$ & 0.0085 \\
MOEA/D-IWO with TE & $\mathbf{0 . 0 0 3 7}$ & $\mathbf{0 . 0 0 5 6}$ & $(\mathbf{0 . 5 1 0 0 , 0 . 4 9 0 0 )}$ & $\mathbf{0 . 0 0 4 6}$ \\
MOEA/D-IWO with WS & 0.0062 & 0.0060 & $(0.5450,0.4550)$ & 0.0061 \\
\hline
\end{tabular}

TABLE 4: The best compromise solutions for the second instance by MOEA/D-IWO and MOEA/D-DE (DRR = 5).

\begin{tabular}{lcccc}
\hline & $f_{3}$ (flat-top beam) & $f_{4}$ (cosecant beam) & $\left(\lambda_{3}, \lambda_{4}\right)$ & Cost $=\lambda_{3} f_{3}+\lambda_{4} f_{4}$ \\
\hline MOEA/D-DE with TE & 0.0043 & 0.0578 & $(0.5200,0.4800)$ & 0.0300 \\
MOEA/D-DE with WS & 0.0762 & 0.1702 & $(0.4400,0.5600)$ & 0.1288 \\
MOEA/D-IWO with TE & $\mathbf{0 . 0 0 1 5}$ & $\mathbf{0 . 0 5 1 1}$ & $(\mathbf{0 . 4 7 5 0 , 0 . 5 2 5 0 )}$ & $\mathbf{0 . 0 2 7 5}$ \\
MOEA/D-IWO with WS & 0.0016 & 0.0516 & $(0.4650,0.5350)$ & 0.0284 \\
\hline
\end{tabular}

maximum values of each objective function together with the rate of increase of membership satisfaction. As in $[33,34]$, we assume that $\mu_{i}$ is a strictly monotonic decreasing and continuous function defined as

$$
\mu_{i}= \begin{cases}1, & \text { if } f_{i} \leq f_{i}^{\min }, \\ \frac{f_{i}^{\max }-f_{i}}{f_{i}^{\max }-f_{i}^{\min },} & \text { if } f_{i}^{\min } \leq f_{i} \leq f_{i}^{\max }, \\ 0, & \text { if } f_{i} \geq f_{i}^{\max },\end{cases}
$$

where $f_{i}^{\max }$ and $f_{i}^{\text {min }}$ are the maximum and minimum values of the $i$ th objective solutions among all nondominated solutions, respectively. Value $\mu_{i}$ indicates how much a nondominated solution satisfies the $i$ th objective, so the sum of $\mu_{i}(i=1,2, \ldots, m)$ for all the objectives can be calculated for measuring the "accomplishment" of each solution in satisfying the $M$ objectives and then a membership function $R_{u}\left(u=1,2, \ldots, N_{s}\right)$ can be defined for nondominated solutions as follows:

$$
R_{u}=\frac{\sum_{i=1}^{M} \mu_{i}(u)}{\sum_{v=1}^{N_{s}} \sum_{i=1}^{M} \mu_{i}(v)},
$$

where $N_{s}$ represents the number of nondominated solutions. The function $R_{u}$ can be treated as a membership function for the nondominated solutions in a fuzzy set. Value $R_{u}$ represents the quality of a solution among all nondominated solutions, and the one with the maximum value of $\mu^{q}$ is the best compromise solution.

6.3. Results. Effectiveness of the MOEA/D-IWO algorithm is verified through the design of two examples. In the optimization process, excitation amplitudes range from 0 to 1 , and phases are restricted from -180 to 180 degrees.

6.3.1. Problem 1. Consider a 20-element $0.5 \lambda$-equispaced symmetrical array with $\operatorname{EF}(\theta)=\sin (\theta)$ for generating a flattop beam and a pencil beam. The flat-top beam is realized by the common excitation amplitudes and unknown phases, and the phases for forming pencil beam are set to zero. Therefore, there are 10 common excitation amplitude variables and 10 phase variables for forming flat-top beam, to be optimized in objective function (17). The maximum dynamic range ratio (DRR) of excitation amplitudes is controlled with 4.

The best compromise solutions obtained by MOEA/DIWO and MOEA/D-DE are shown in Figure 1 and Table 1. It can be seen that, for flat-top beam, the peak SLL obtained by MOEA/D-IWO is $0.67 \mathrm{~dB}$ lower than that obtained by MOEA/D-DE. The BW value obtained by MOEA/D-DE is $1.6^{\circ}$ wider than the objective value, while that obtained by MOEA/D-IWO is just $0.2^{\circ}$ wider than the design objective. For the pencil beam, the pattern obtained by MOEA/D-IWO has $0.94 \mathrm{~dB}$ lower peak SLL value, when other indexes meet the design objectives well. The excitation amplitudes and the phases for the synthesized radiation patterns obtained by MOEA/D-IWO are shown in Figure 2.

6.3.2. Problem 2. Consider a $0.5 \lambda$-equispaced linear array with 25 isotropic elements to generate a flat-top beam and a cosecant-squared beam. The dimension of vector $x$ in objective function (20) is 75 , including 25 common amplitudes and 50 unknown phases. The maximum DRR of excitation amplitudes is controlled with $\mathrm{DRR}=5$.

Radiation patterns of the best compromise solutions obtained by MOEA/D-IWO and MOEA/D-DE are shown in Figure 4 and Table 2, and the excitation amplitudes and the phases for the synthesized radiation patterns obtained by MOEA/D-IWO are shown in Figure 5. It can be seen from Table 2 that, for the flat-top beam, the ripples obtained by these two algorithms meet the design objectives well, the peak SLL value obtained by MOEA/D-IWO is $0.9553 \mathrm{~dB}$ lower than that obtained by MOEA/D-DE, and the BW values obtained by MOEA/D-DE and MOEA/D-IWO are 0.88 and 0.86 , respectively. For the cosecant beam, the BW obtained by MOEA/D-IWO is smaller than that obtained by MOEA/D-DE, and the ripple and peak SLL values obtained by MOEA/D-IWO are $0.3247 \mathrm{~dB}$ and $0.8325 \mathrm{~dB}$ lower than those obtained by MOEA/D-DE, respectively. 


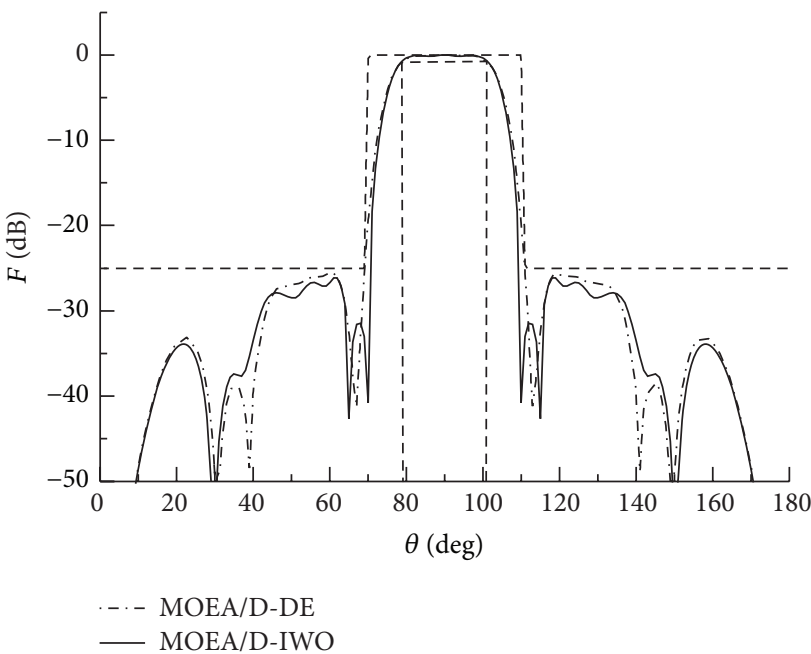

(a)

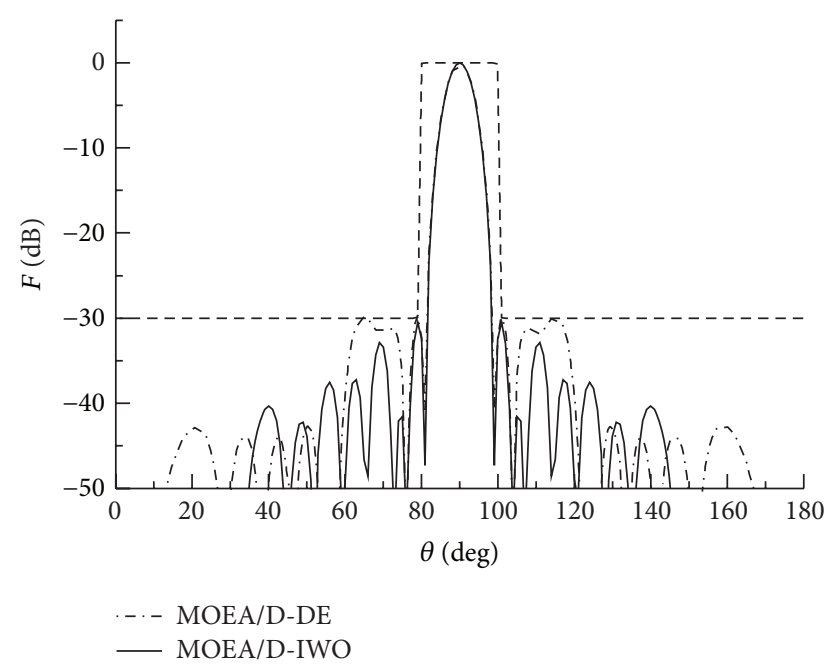

(b)

FIGURE 1: Synthesized radiation patterns and their masks (dashed black lines) for Problem 1: (a) flat-top beam and (b) pencil beam.

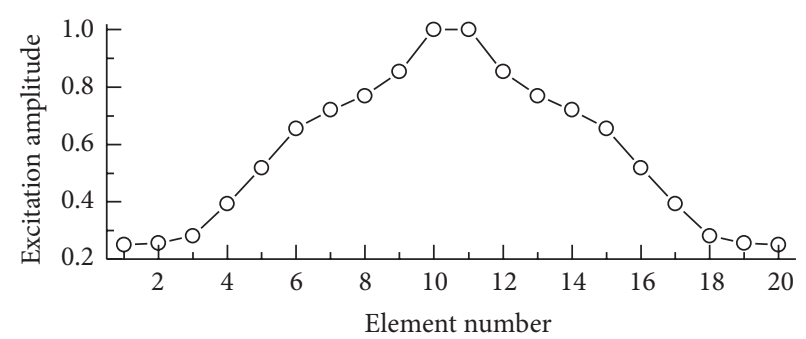

(a)

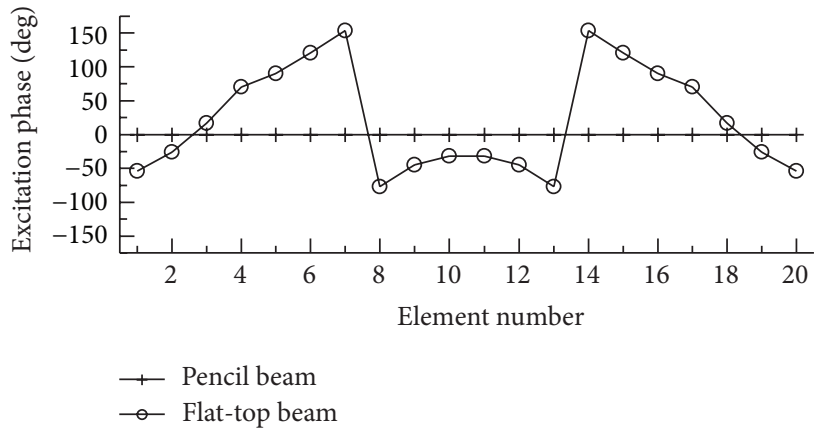

(b)

FIGURE 2: Excitation coefficients for the synthesized radiation patterns obtained by MOEAD-IWO for Problem 1: (a) common amplitudes and (b) different phases.

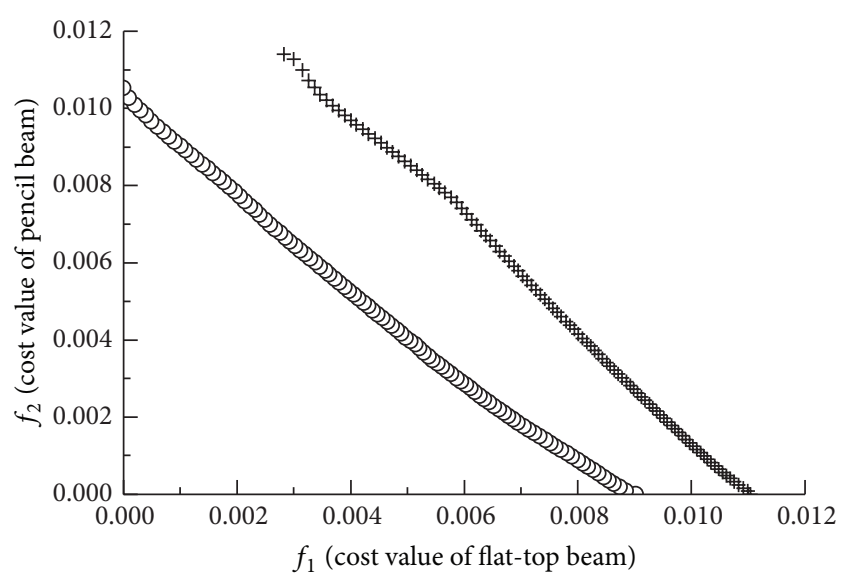

+ MOEA/D-DE

- MOEA/D-IWO

FIGURE 3: Approximate PFs obtained by MOEA/D-DE and MOEA/D-IWO for Problem 1.
Thus, we conclude that the patterns obtained by MOEA/DIWO have better performance. The advantage of MOEA/DIWO over MOEA/D-DE can also be seen from the obtained approximate PFs shown in Figures 3 and 6.

6.3.3. Comparisons with Different Decomposition Approaches. In order to make further comparisons between MOEA/DIWO and MOEA/D-DE, weighted sum method [23] is also used in the two algorithms to optimize Problem 1 and Problem 2. Related parameters are set the same as in Section 6.1. All the algorithms run 20 times independently, and the best results are compared.

Tables 3 and 4 show the best compromise solutions obtained by MOEA/D-IWO and MOEA/D-DE for Problem 1 and Problem 2, respectively, in which TE and WS are the abbreviations of Tchebycheff approach and weighted sum. $f_{1}$ and $f_{2}$ are the cost values of flat-top beam and pencil beam in Problem 1; $f_{3}$ and $f_{4}$ are the cost values of flat-top beam and cosecant beam in Problem $2 .\left(\lambda_{1}, \lambda_{2}\right)$ and $\left(\lambda_{3}, \lambda_{4}\right)$ are 


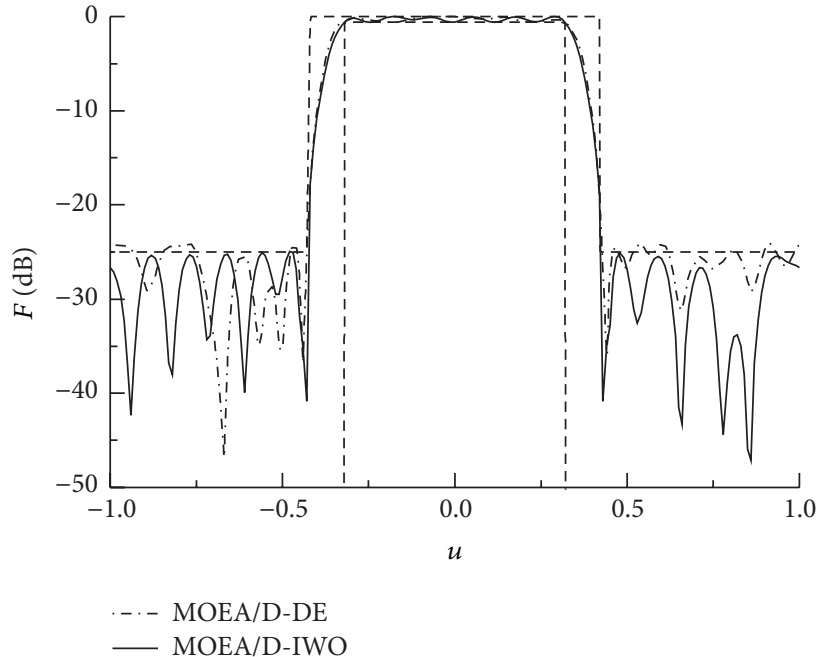

(a)

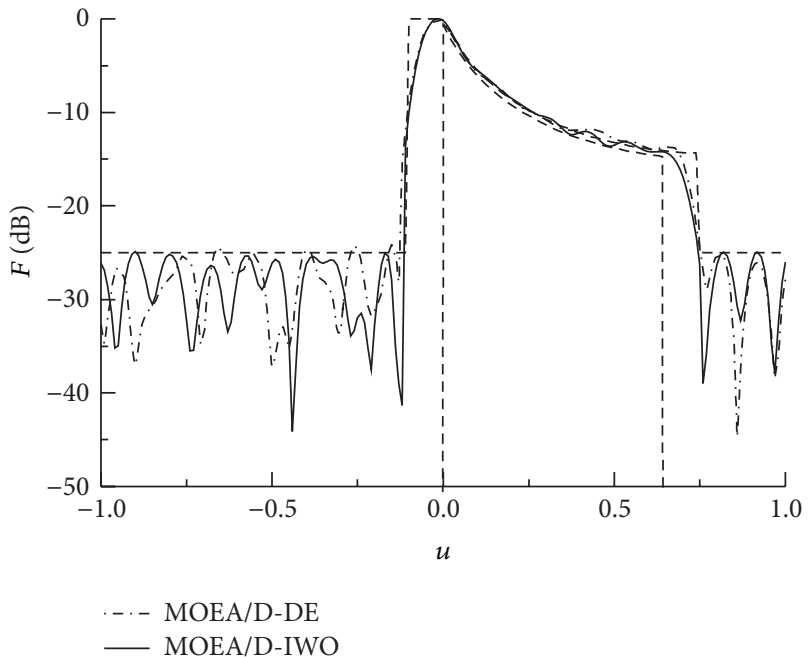

(b)

FIGURE 4: Synthesized radiation patterns and their masks (dashed black lines) for Problem 2: (a) flat-top beam and (b) cosecant-squared beam.

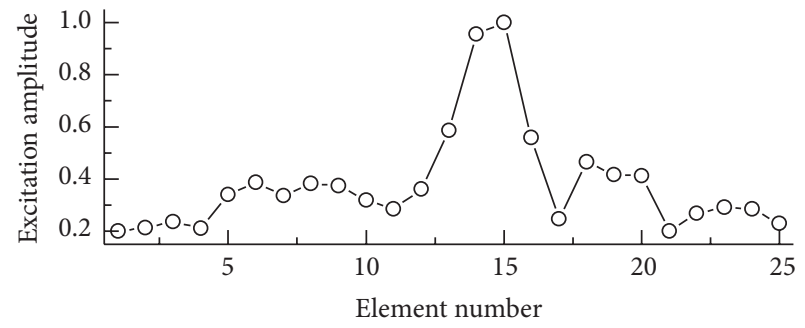

(a)

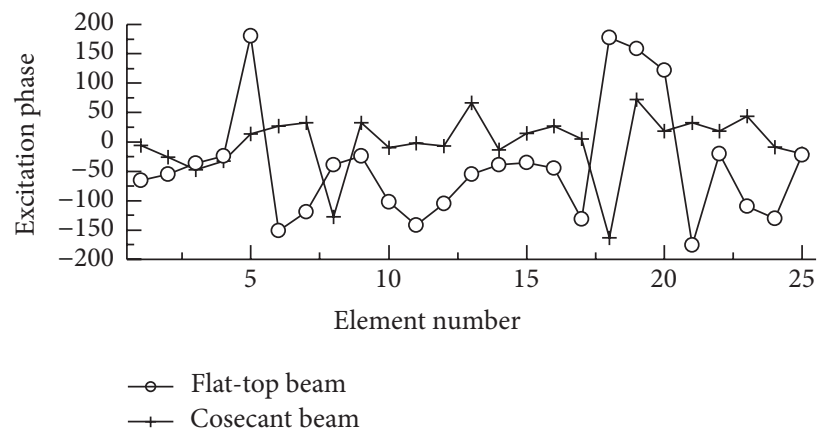

(b)

FIGURE 5: Excitation coefficients for the synthesized radiation pattern obtained by MOEAD-IWO for Problem 2: (a) common amplitudes and (b) different phases.

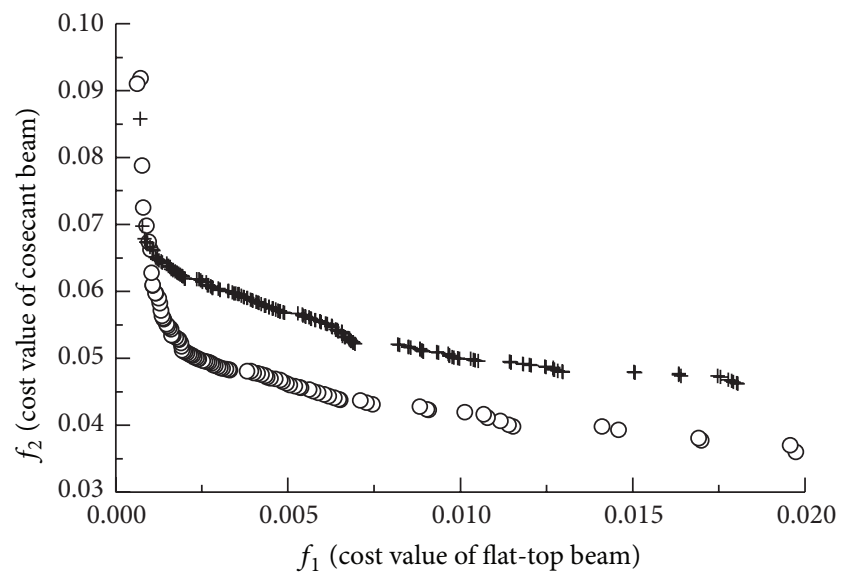

+ MOEA/D-DE

- MOEA/D-IWO

FIgURe 6: The approximate PFs obtained by MOEA/D-DE and MOEA/D-IWO for Problem 2. the weight vectors for the compromise solutions. The overall costs are computed by $\lambda_{1} f_{1}+\lambda_{2} f_{2}$ and $\lambda_{3} f_{3}+\lambda_{4} f_{4}$. Best values are shown in bold. We can conclude that MOEA/D-IWO with Tchebycheff approach can obtain better compromise solutions than other algorithms.

Tables 5 and 6 present the comparison of the three metrics obtained in the two experiments; best values are shown in bold. It is obvious that, when using Tchebycheff approach as the decomposition approach, MOEA/DIWO has higher accuracy ( $D$-metric), better uniformity $(\Delta$ metric), and larger extent ( $\nabla$-metric) than MOEA/D-DE. Among the four algorithms, MOEA/D-DE with weighted sum shows the best mean, maximum, and minimum values of $\nabla$-metric. MOEA/D-IWO with Tchebycheff approach performs better than the other three algorithms in terms of $D$-metric, $\Delta$-metric, and the standard deviation of $\nabla$ metric. Comparison between the four algorithms reveals the superiority of MOEA/D-IWO and confirms its potential for array synthesis problems. 
TABLE 5: Performance comparison of MOEA/D-DE and MOEA/D-IWO with different decomposition approaches for Problem 1.

\begin{tabular}{|c|c|c|c|c|}
\hline Algorithm & Mean & Std & Max & Min \\
\hline & \multicolumn{4}{|c|}{$D$-metric } \\
\hline MOEA/D-DE with TE & $8.97 e-3$ & $5.47 e-3$ & $9.27 e-3$ & $6.54 e-3$ \\
\hline MOEA/D-DE with WS & $9.12 e-3$ & $5.34 e-3$ & $9.64 e-3$ & $7.35 e-3$ \\
\hline MOEA/D-IWO with TE & $5.89 e-3$ & $3.27 e-3$ & $6.84 e-3$ & $4.31 \mathrm{e}-3$ \\
\hline \multirow[t]{2}{*}{ MOEA/D-IWO with WS } & $5.92 e-3$ & $3.35 e-3$ & $6.91 e-3$ & $5.39 e-3$ \\
\hline & \multicolumn{4}{|c|}{$\Delta$-metric } \\
\hline MOEA/D-DE with TE & $2.14 e-3$ & $1.21 e-3$ & $3.07 e-3$ & $1.65 e-3$ \\
\hline MOEA/D-DE with WS & $2.04 e-3$ & $1.02 e-3$ & $2.98 e-3$ & $1.32 e-3$ \\
\hline MOEA/D-IWO with TE & $5.14 e-4$ & $3.17 e-4$ & $6.12 e-4$ & $3.87 e-4$ \\
\hline \multirow[t]{2}{*}{ MOEA/D-IWO with WS } & $5.23 e-4$ & $3.24 e-4$ & $6.35 e-4$ & $3.97 e-4$ \\
\hline & \multicolumn{4}{|c|}{$\nabla$-metric } \\
\hline MOEA/D-DE with TE & $9.05 e-5$ & $5.24 e-5$ & $9.36 e-5$ & $8.79 e-5$ \\
\hline MOEA/D-DE with WS & $2.89 e-4$ & $1.02 e-5$ & $3.02 \mathrm{e}-4$ & $2.39 e-4$ \\
\hline MOEA/D-IWO with TE & $9.34 e-5$ & $2.14 e-6$ & $9.48 e-5$ & $9.31 e-5$ \\
\hline MOEA/D-IWO with WS & $1.61 e-4$ & $8.32 e-6$ & $1.64 e-4$ & $1.51 e-4$ \\
\hline
\end{tabular}

TABLE 6: Performance comparison of MOEA/D-DE and MOEA/D-IWO with different decomposition approaches for Problem 2.

\begin{tabular}{|c|c|c|c|c|}
\hline Algorithm & Mean & Std & Max & Min \\
\hline & \multicolumn{4}{|c|}{$D$-metric } \\
\hline MOEA/D-DE with TE & $5.31 e-2$ & $2.39 e-2$ & $7.25 e-2$ & $4.21 e-2$ \\
\hline MOEA/D-DE with WS & $6.21 e-2$ & $4.52 e-2$ & $7.95 e-2$ & $5.31 e-2$ \\
\hline MOEA/D-IWO with TE & $3.58 e-2$ & $1.54 \mathrm{e}-2$ & $4.31 \mathrm{e}-2$ & $3.01 \mathrm{e}-2$ \\
\hline \multirow[t]{2}{*}{ MOEA/D-IWO with WS } & $4.91 e-2$ & $2.31 e-2$ & $6.12 e-2$ & $3.95 e-2$ \\
\hline & \multicolumn{4}{|c|}{$\Delta$-metric } \\
\hline MOEA/D-DE with TE & $3.87 e-2$ & $1.35 e-2$ & $4.59 e-2$ & $2.74 e-2$ \\
\hline MOEA/D-DE with WS & $6.98 e-2$ & $5.32 e-2$ & $7.25 e-2$ & $5.35 e-2$ \\
\hline MOEA/D-IWO with TE & $1.78 \mathrm{e}-2$ & $1.01 \mathrm{e}-2$ & $2.14 \mathrm{e}-2$ & $1.21 \mathrm{e}-2$ \\
\hline \multirow[t]{2}{*}{ MOEA/D-IWO with WS } & $2.32 e-2$ & $1.29 e-2$ & $4.02 e-2$ & $2.54 e-2$ \\
\hline & \multicolumn{4}{|c|}{$\nabla$-metric } \\
\hline MOEA/D-DE with TE & $6.70 e-4$ & $4.27 e-4$ & $6.85 e-4$ & $5.87 e-4$ \\
\hline MOEA/D-DE with WS & $2.30 e-2$ & $3.29 e-4$ & $2.43 e-2$ & $2.01 \mathrm{e}-2$ \\
\hline MOEA/D-IWO with TE & $1.02 e-3$ & $3.24 e-5$ & $1.10 e-3$ & $1.02 e-3$ \\
\hline MOEA/D-IWO with WS & $1.23 e-2$ & $1.23 e-4$ & $1.34 e-2$ & $1.17 e-2$ \\
\hline
\end{tabular}

\section{Conclusion}

In this paper, synthesis of phase-only reconfigurable antenna array is formulated as an MOP where the design objectives are the different patterns. We integrate the modified IWO algorithm in MOEA/D and propose a new version of MOEA/D, called MOEA/D-IWO, for solving the multiobjective optimization problems. The new algorithm makes good use of the powerful searching and colonizing ability of invasive weeds and maintains the advantages of the original MOEA/D. Two phase-only reconfigurable antenna array examples are synthesized by the proposed algorithm, and the results have been compared with those obtained by the existing MOEA/D$\mathrm{DE}$ algorithm. The comparing simulation results indicate the superiority of the proposed algorithm over MOEA/D$\mathrm{DE}$ in synthesizing the phase-only reconfigurable antenna array. The proposed algorithm opens a new prospect in array synthesis problems. It may also be applied in other challenging fields of MOPs for future research.

\section{Conflict of Interests}

The authors declare that there is no conflict of interests regarding the publication of this paper.

\section{Acknowledgment}

This research was fully supported by the National Natural Science Foundation of China (no. 61201022).

\section{References}

[1] O. M. Bucci, G. Mazzarella, and G. Panariello, "Reconfigurable arrays by phase-only control," IEEE Transactions on Antennas and Propagation, vol. 39, no. 7, pp. 919-925, 1991.

[2] R. Vescovo, "Reconfigurability and beam scanning with phaseonly control for antenna arrays," IEEE Transactions on Antennas and Propagation, vol. 56, no. 6, pp. 1555-1565, 2008.

[3] A. F. Morabito, A. Massa, P. Rocca, and T. Isernia, "An effective approach to the synthesis of phase-only reconfigurable linear 
arrays," IEEE Transactions on Antennas and Propagation, vol. 60, no. 8, pp. 3622-3631, 2012.

[4] S. Baskar, A. Alphones, and P. N. Suganthan, "Geneticalgorithm-based design of a reconfigurable antenna array with discrete phase shifters," Microwave and Optical Technology Letters, vol. 45, no. 6, pp. 461-465, 2005.

[5] G. K. Mahanti, A. Chakrabarty, and S. Das, "Phase-only and amplitude-phase synthesis of dual-pattern linear antenna arrays using floating-point genetic algorithms," Progress in Electromagnetics Research, vol. 68, pp. 247-259, 2007.

[6] D. W. Boeringer and D. H. Werner, "Particle swarm optimization versus genetic algorithms for phased array synthesis," IEEE Transactions on Antennas and Propagation, vol. 52, no. 3, pp. 771-779, 2004.

[7] D. Gies and Y. Rahmat-Samii, "Particle swarm optimization for reconfigurable phase-differentiated array design," Microwave and Optical Technology Letters, vol. 38, no. 3, pp. 168-175, 2003.

[8] D. Gies and Y. Rahmat-Samii, "Reconfigurable array design using parallel particle swarm optimization," in Proceedings of the IEEE International Antennas and Propagation Symposium and USNC/CNC/URSI North American Radio Science Meeting, vol. 1, pp. 177-180, June 2003.

[9] X. Li and M. Yin, "Hybrid differential evolution with biogeography-based optimization for design of a reconfigurable antenna array with discrete phase shifters," International Journal of Antennas and Propagation, vol. 2011, Article ID 685629, 12 pages, 2011.

[10] X. Li and M. Yin, "Design of a reconfigurable antenna array with discrete phase shifters using differential evolution algorithm," Progress In Electromagnetics Research B, vol. 31, pp. 29-43, 2011.

[11] Q. Zhang and H. Li, "MOEA/D: a multiobjective evolutionary algorithm based on decomposition," IEEE Transactions on Evolutionary Computation, vol. 11, no. 6, pp. 712-731, 2007.

[12] H. Li and Q. Zhang, "Multiobjective optimization problems with complicated pareto sets, MOEA/D and NSGA-II," IEEE Transactions on Evolutionary Computation, vol. 13, no. 2, pp. 284-302, 2009.

[13] Q. Zhang, W. Liu, and H. Li, "The performance of a new version of MOEA/D on CEC09 unconstrained MOP test instances," in Proceeding of the IEEE Congress on Evolutionary Computation (CEC '09), pp. 203-208, Trondheim, Norway, May 2009.

[14] Q. Zhang, W. Liu, E. Tsang, and B. Virginas, "Expensive multiobjective optimization by MOEA/D with gaussian process model," IEEE Transactions on Evolutionary Computation, vol. 14, no. 3, pp. 456-474, 2010.

[15] A. R. Mehrabian and C. Lucas, "A novel numerical optimization algorithm inspired from weed colonization," Ecological Informatics, vol. 1, no. 4, pp. 355-366, 2006.

[16] S. Karimkashi and A. A. Kishk, "Invasive weed optimization and its features in electromagnetics," IEEE Transactions on Antennas and Propagation, vol. 58, no. 4, pp. 1269-1278, 2010.

[17] G. G. Roy, S. Das, P. Chakraborty, and P. N. Suganthan, "Design of non-uniform circular antenna arrays using a modified invasive weed optimization algorithm," IEEE Transactions on Antennas and Propagation, vol. 59, no. 1, pp. 110-118, 2011.

[18] Z. D. Zaharis, C. Skeberis, and T. D. Xenos, "Improved antenna array adaptive beam-forming with low side lobe level using a novel adaptive invasive weed optimization method," Progress in Electromagnetics Research, vol. 124, pp. 137-150, 2012.

[19] A. R. Mallahzadeh, H. Oraizi, and Z. Davoodi-Rad, "Application of the invasive weed optimization technique for antenna configurations," Progress in Electromagnetics Research, vol. 79, pp. 137-150, 2008.

[20] A. R. Mallahzadeh, S. Es'haghi, and H. R. Hassani, "Compact U-array MIMO antenna designs using IWO algorithm," International Journal of $R F$ and Microwave Computer-Aided Engineering, vol. 19, no. 5, pp. 568-576, 2009.

[21] D. Kundu, K. Suresh, S. Ghosh, S. Das, and B. K. Panigrahi, "Multi-objective optimization with artificial weed colonies," Information Sciences, vol. 181, no. 12, pp. 2441-2454, 2011.

[22] H. Scheffe, "Experiments with mixtures," Journal of the Royal Statistical Society B: Methodological, vol. 20, pp. 344-360, 1958.

[23] K. Miettinen, Nonlinear Multiobjective Optimization, Kluwer Academic Publishers, Norwell, Mass, USA, 1999.

[24] H. Sato, "Inverted PBI in MOEA/D and its impact on the search performance on multi and many-objective optimization," in Proceedings of the 2014 Conference on Genetic and Evolutionary Computation, pp. 645-652, New York, NY, USA, 2014.

[25] D. V. Veldhuizen, Multiobjective evolutionary algorithms: classifications, analyses, and new innovations [Ph.D. thesis], Department of Electrical and Computer Engineering, Air Force Institute of Technology, Wright-Patterson AFB, Ohio, USA, 1999.

[26] E. Zitzler, L. Thiele, M. Laumanns, C. M. Fonseca, and V. G. da Fonseca, "Performance assessment of multiobjective optimizers: an analysis and review," IEEE Transactions on Evolutionary Computation, vol. 7, no. 2, pp. 117-132, 2003.

[27] K. Deb, A. Pratap, S. Agarwal, and T. Meyarivan, "A fast and elitist multiobjective genetic algorithm: NSGA-II," IEEE Transactions on Evolutionary Computation, vol. 6, no. 2, pp. 182197, 2002.

[28] K. Deb, Multi-Objective Optimization Using Evolutionary Algorithms, John Wiley \& Sons, Chichester, UK, 2001.

[29] C. Erbas, S. Cerav-Erbas, and A. D. Pimentel, "Multiobjective optimization and evolutionary algorithms for the application mapping problem in multiprocessor system-on-chip design," IEEE Transactions on Evolutionary Computation, vol. 10, no. 3, pp. 358-374, 2006.

[30] Y. Y. Tan, Y. C. Jiao, H. Li, and X. K. Wang, "A modification to MOEA/D-DE for multiobjective optimization problems with complicated Pareto sets," Information Sciences, vol. 213, pp. 1438, 2012.

[31] http://cswww.essex.ac.uk/staff/qzhang.

[32] C. G. Tapia and B. A. Murtagh, "Interactive fuzzy programming with preference criteria in multiobjective decision-making," Computers \& Operations Research, vol. 18, no. 3, pp. 307-316, 1991.

[33] J. S. Dhillon, S. C. Parti, and D. P. Kothari, "Stochastic economic emission load dispatch," Electric Power Systems Research, vol. 26, no. 3, pp. 179-186, 1993.

[34] S. Pal, B. Qu, S. Das, and P. N. Suganthan, "Linear antenna array synthesis with constrained multi-objective differential evolution," Progress in Electromagnetics Research B, vol. 21, pp. 87-111, 2010. 

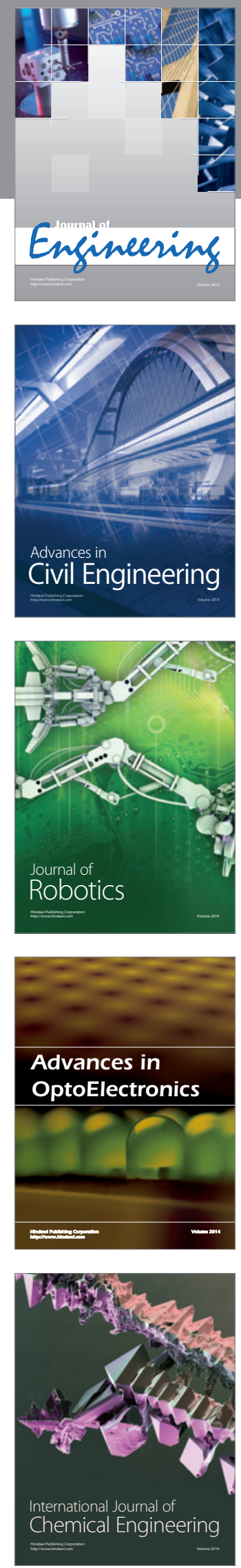

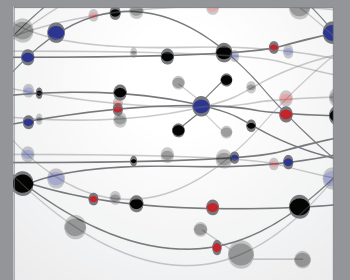

The Scientific World Journal
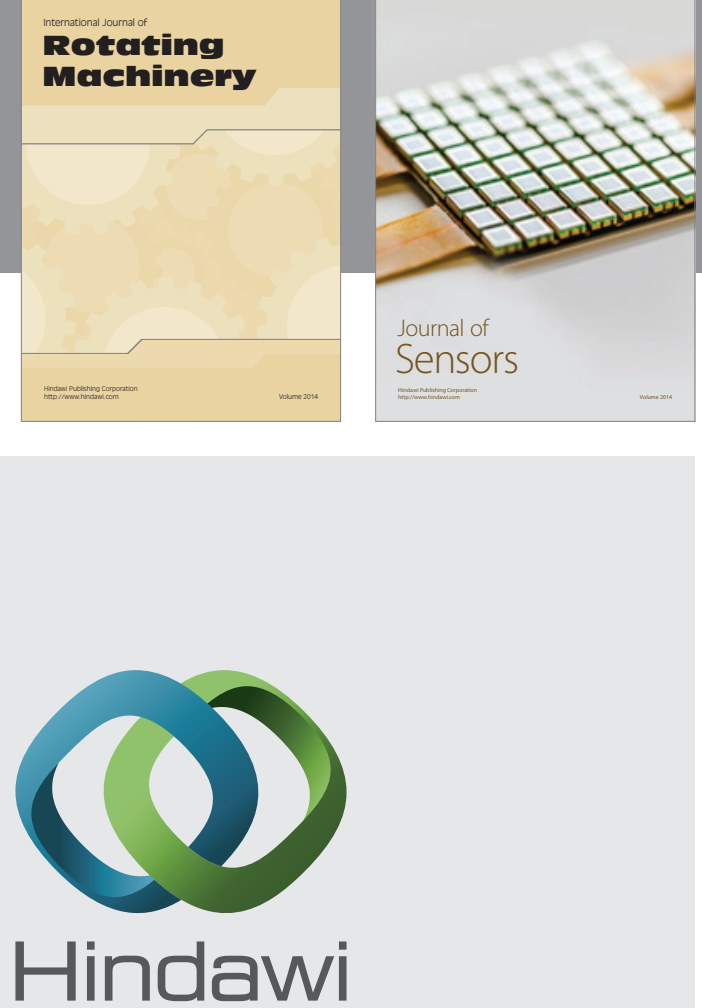

Submit your manuscripts at http://www.hindawi.com
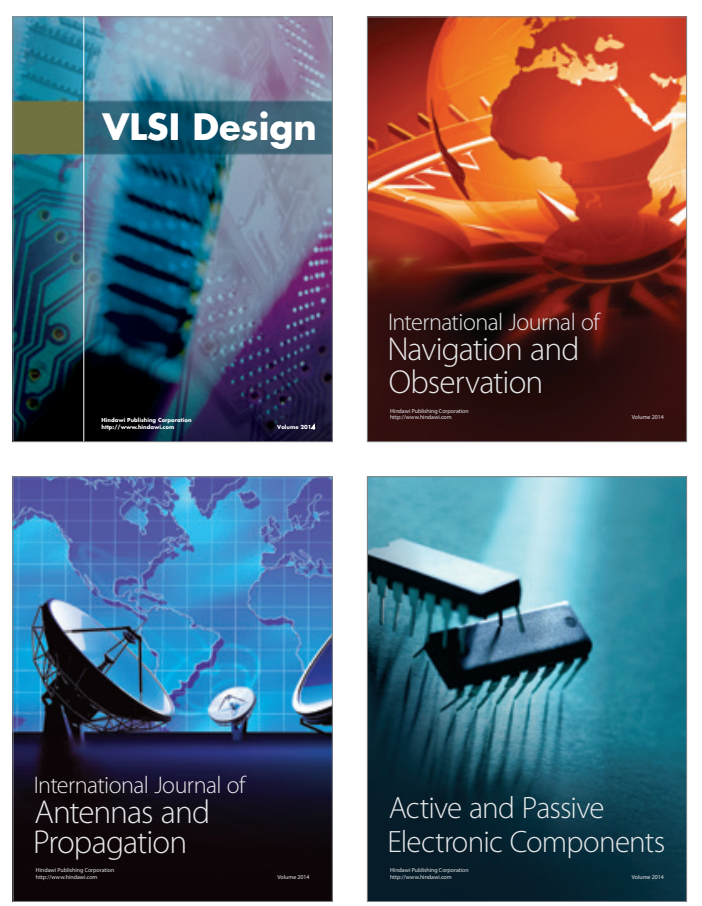
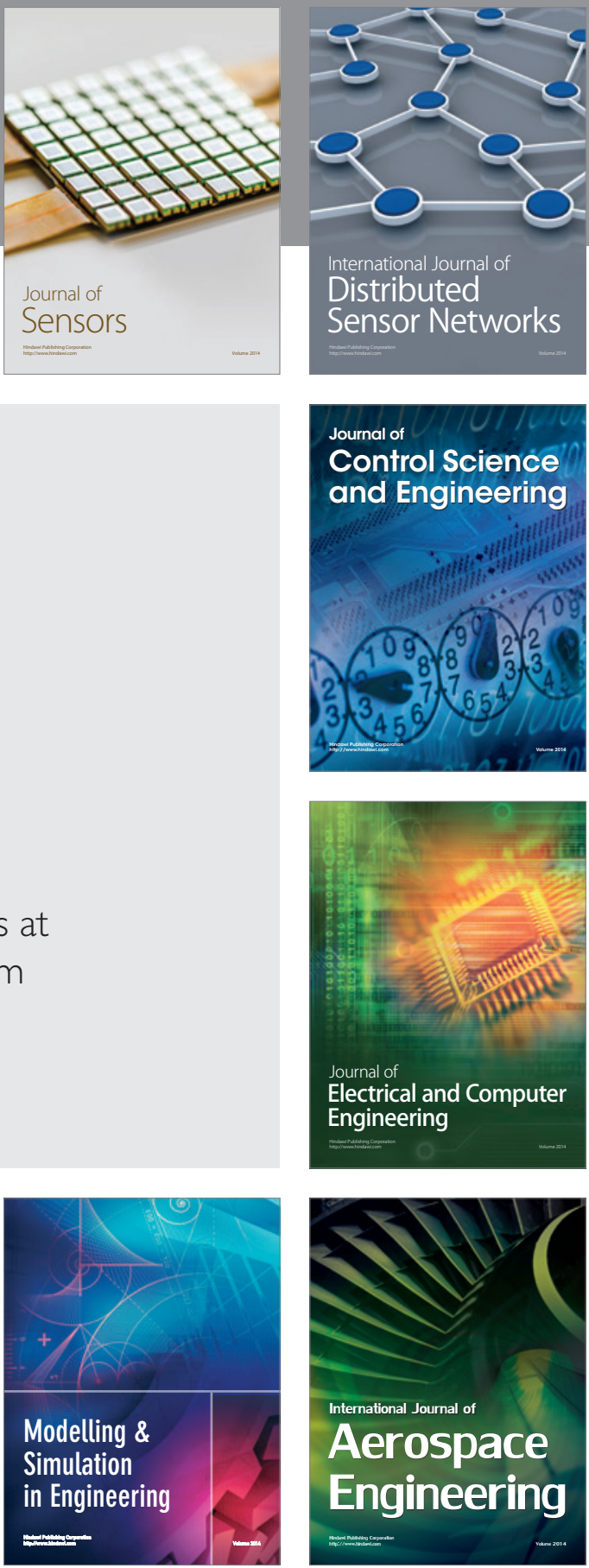

Journal of

Control Science

and Engineering
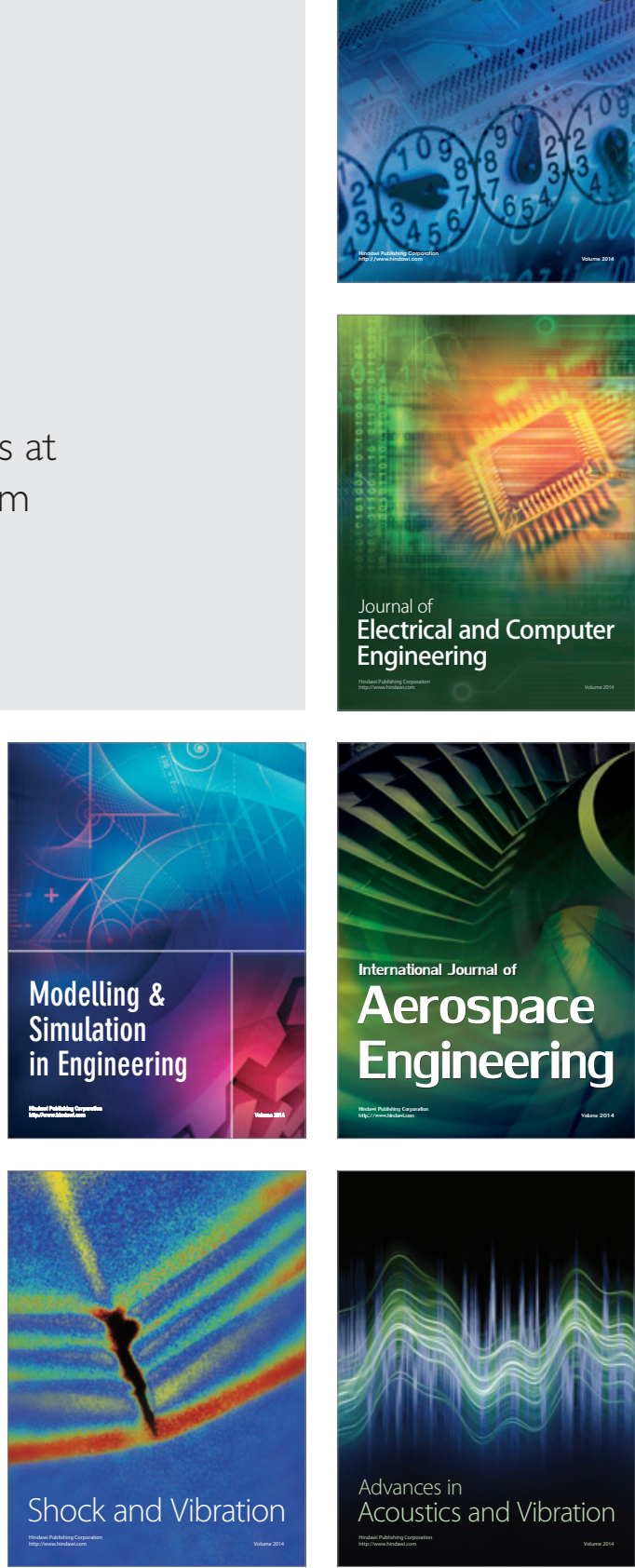